\title{
Joint lot sizing and tool management in a CNC environment
}

\author{
M. Selim Akturk *, Siraceddin Onen \\ Department of Industrial Engineering, Bilkent University, 06533 Bilkent, Ankara, Turkey
}

Received 25 March 1997; accepted 9 November 1998

\begin{abstract}
We propose a new algorithm to solve lot sizing, tool allocation and machining conditions optimization problems simultaneously to minimize total production cost in a CNC environment. Most of the existing lot sizing and tool management methods solve these problems independently using a two-level optimization approach. Thus, we not only improve the overall solution by exploiting the interactions among these decision making problems, but also prevent any infeasibility that might occur for the tool management problem due to decisions made at the lot sizing level. The computational experiments showed that in a set of randomly generated problems $22.5 \%$ of solutions found by the two-level approach were infeasible and the proposed joint approach improved the solution on the average by $6.79 \%$ for the remaining cases. (C) 1999 Elsevier Science B.V. All rights reserved.
\end{abstract}

Keywords: Computer numerical control; Flexible manufacturing systems; Lot sizing; Tool management

\section{Introduction}

In view of the high investment and operating costs of computer numerically controlled machines (CNCs) and hence of flexible manufacturing systems (FMSs) attention should be paid to their effective utilization. Gray et al. [6] and Veeramani et al. [18] give extensive surveys on the tool management issues of automated manufacturing systems, and emphasize that the lack of tooling considerations has resulted in the poor performance of these systems. Kouvelis [11] identified cutting tool utilization as an important parameter for the overall system performance. In this study, the cost of tooling has been reported to be $25-30 \%$ of the fixed and variable cost

\footnotetext{
* Corresponding author. Tel.: +90-312-266-4477; fax: +90312-266-4126; e-mail: akturk@bilkent.edu.tr
}

of production. Gray et al. [6] present an integrated conceptual framework for resource planning to examine how tool management issues can be classified into tool, machine and system levels, and point out that efforts in tool management focused on single level decisions.

Most of the existing studies in tool management ignore the lot sizing decision at system level and take it as a predetermined input while deciding on tool allocation and machining parameters. On the other hand, most of the lot sizing models treat the production rates either as fixed or infinite to determine the lot size for each item independently by minimizing the sum of setup and inventory holding costs, while in practice the production rates are significant decision variables. In an automated manufacturing environment, operational problems, such as machining conditions, tool availability and tool life, 
should be taken into account for the reliable modeling of CNCs, or the absence of such crucial constraints may lead to infeasible results. Consequently, total production cost can be decreased and any infeasibility due to machine capacity limitation can be avoided by controlling production rates.

The purpose of this paper is to evaluate the efficacy of an integrated approach of solving the lot sizing and tool management problems simultaneously. Using a two-level approach, the operating decisions for the lot sizing problem are determined relatively independent of the operating decisions for the tool management problem. Therefore, in a twolevel approach, a decision made at a higher level without considering its impact on the lower level can lead to infeasible or inferior results, because the constraints and/or costs at the lower level are not fully reflected in the higher level problem. Historically this is the manner in which many organizations arrive at these decisions. It is certainly clear that the existing decomposition is sub-optimal. By combining both decision making problems into one, some economic advantage may be obtained over solving these problems separately.

For solving the tool allocation problem at the system level, most of the published studies use $0-1$ binary variables, i.e., a particular tool $j$ is assigned to operation $i$, to represent tool requirements. Stecke [14] formulates the FMS loading problem as a nonlinear mixed integer programming (MIP) problem and solves it through linearization techniques. Sodhi et al. [13] propose a four level hierarchy for production control of FMSs, including part type selection and loading, and present various models at each level. Sarin and Chen [12] give an MIP formulation under the assumption that the total machining costs depend upon the tool-machine combination. The tool life is considered as a constraint in the formulation. Unfortunately, all of these studies assume constant lot sizes, production rates as well as processing times. Furthermore, these studies determine the tool requirements for each operation independently, and fail to relate the contention among the operations for a limited number of tools.

At the machine level, there exist several studies paying attention to tooling issues like tool selection, tool magazine loading and minimization of tool switches due to a change in a part mix, at both the long term planning and operational level (Kouvelis [11]; Tang and Denardo [16]). Unfortunately, these studies also assume constant lot sizes, processing times and tool lives, even though the tool replacement frequency is directly related with the machining conditions selections. Further, in the multiple operation case, non-machining time components, such as the tool replacements due to tool wear, can have a significant impact on the total cost of production and the throughput of parts as shown by Tetzlaff [17]. Gray et al. [6] reported that tools are changed ten times more often due to tool wear than to part mix because of the relatively short tool lives of many turning tools. The machining conditions optimization for a single operation is a well known problem, where the decision variables are the cutting speed and feed rate. Several models and solution methodologies have been developed in the literature (Gopalakrishnan and Al-Khayyal [4]; Tan and Creese [15]). However, these models consider only the contribution of machining time and tooling cost to the total cost of operation, usually ignoring the contribution of non-machining time components to the operating cost, which could be very significant for the multiple operation case. Further, these studies exclude the tooling issues such as tool availability and tool life capacity limitations.

In the literature there exist few studies on the integration of lot sizing and tool management problems. Wysk et al. [19] introduce lot size considerations in determining the optimal cutting speed in a single item, single machine problem. Koulamas [9] presents a queueing model for determining analytically the optimal lot size in a machining economics problem under stochastic tool life considerations. Koulamas [10] proposes an iterative procedure for the simultaneous determination of the cutting speed and lot size values on a single machine for single and multiple part cases using the Lagrangian technique, while the feed rate is taken as a constant. In this study, parts are assumed to be composed of a single operation. Consequently, parts are machined by a single cutting tool and tool allocation decisions are not considered. The author also has not considered machine horsepower, surface finish and tool availability constraints, although in many real life problems the machining parameters are constrained by these limitations. 
We propose a new solution methodology to find optimal lot sizes, tool allocations and machining parameters by integrating system, machine and tool level decisions for production of multiple parts consisting of multiple operations in a CNC environment. The importance of these concepts has been mentioned at different decision making levels. We identified the need for such a problem formulation especially for computer aided process planning and part programming. This study can be considered as a module in the framework of a fully integrated system (Kalta and Davies [7] and Karadkar and Pande [8]). The remainder of this paper is organized into six sections as follows. In Section 2, we discuss the underlying assumptions and introduce a mathematical model of the problem. The proposed algorithm is described in Section 3. A numerical example and the computational results of an experimental design are presented in Sections 4 and 5, respectively. Finally, some concluding remarks are provided in Section 6.

\section{Mathematical model}

The notation used in the proposed mathematical model is as follows:

Parameters
$\alpha_{j}, \beta_{j}, \gamma_{j}$
$C_{m}, b, c, e$
$C_{o}$
$C_{s}, g, h, l$
$C_{t_{j}}$
$d_{i p}$
$D_{p}$
$G_{i p}$
$h_{p}$
$\mathrm{HP}$
$I_{p}$

Speed, feed, depth of cut exponents for tool $j$ Specific coefficient and exponents of the machine power constraint Operating cost of the $\mathrm{CNC}$ machine, $(\$ / \mathrm{min})$

Specific coefficient and exponents of the surface roughness constraint Cost of tool $j,(\$ /$ tool $)$ Depth of cut for operation $i$ of part $p$, (in.)

Demand for part $p$, (parts)

Diameter of the generated surface for $\quad U_{i j p}$ operation $i$ of part $p$, (in.)

Inventory holding cost of part $p, \quad v_{i j p}$ (\$/part/period)

Maximum available machine power, $\quad x_{i j p}$ (hp)

Set of all operations of part $p$
$J$

$J_{i p}$

$L_{i p}$

M

$\mathrm{MH}$

$N_{j}$

$P$

$\mathrm{SF}_{i p}$

$S_{p}$

$\mathrm{TC}_{j}$

$t_{l_{j}}$

$t_{r_{j}}$
$t s_{p}$

\section{Decision variables}

$f_{i j p}$

$n_{i j p}$

$q_{i j p}$

$Q_{1}$

$Q_{2 p}$

$r_{p}$

$s_{p}$

$t_{m_{i j p}}$

$T_{i j p}$

Set of the available tool types

Set of the candidate tool types that can be used for operation $i$ of part $p$ Length of the generated surface for operation $i$ of part $p$, (in.)

A very large positive number, i.e., $M \geq \max \left\{D_{p}\right\}$ for every $p \in P$

Maximum available machine capacity for production of all parts, (min) Number of available tools of type $j$ Set of all parts

Maximum allowable surface roughness for the operation $i$ of part $\mathrm{p}$, ( $\mu$ in.)

Setup cost for production of part $p$, (\$/lot)

Taylor's tool life constant for tool $j$ Tool magazine loading time for a single tool $j,(\min )$

Tool replacing time for tool $j,(\mathrm{~min})$ Setup time for production of part $p$, (min/lot) 
The additional notation used in the proposed algorithm in Section 3 is as follows:

$C_{k p}^{1}, C_{k p}^{2}$
$C_{k p}$
$F$
$H_{k p}^{1}, H_{k p}^{2}$
$H_{k p}$
$K_{p}$
$Q_{1_{k p}}$
$Q_{2_{k p}}$
$r_{k p}$
$R_{j k p}^{1}, R_{j k p}^{2}$
$R_{j k p}$
$y_{i j p}$
$z_{k p}$

Total cost of machining, non-machining and tooling for the lot sizes $Q_{1_{k p}}$ and $Q_{2_{k p}}$, respectively for alternative $k$ of part $p(\$ /$ lot $)$

Total production cost for alternative $k$ of part $p(\$ /$ period $)$

Set of possible equal lots

Total machining and non-machining time required for the lot sizes, $Q_{1_{k p}}$ and $Q_{2_{k p}}$, respectively for alternative $k$ of part $p$ (min/lot)

Total time required for alternative $k$ of part $p$ (min/period)

Set of all lot sizing alternatives of part $p$

Size of equal lots for alternative $k$ of part $p$, (parts)

Size of last lot for alternative $k$ of part $p$, (parts)

Number of equal lots for alternative $k$ of part $p$

Tool type $j$ requirement for the lot sizes, $Q_{1_{k p}}$ and $Q_{2_{k p}}$, respectively for alternative $k$ of part $p$

Total tool type $j$ requirement for alternative $k$ of part $p$

$0-1$ binary indicator which is equal to 1 , if tool $j$ is a candidate tool for operation $i$ of part $p$

0-1 binary decision variable which is equal to 1 , if alternative $k$ of part $p$ is selected

Consider an automated machining environment consisting of a single CNC turning machine. The following assumptions are made to define the scope of this study. There are multiple parts in demanded quantities and each part is composed of multiple operations. Each operation can be performed by a set of alternative tool types from a variety of available tool types with limited quantities on hand. Backlogging is not allowed, and initial and final inventory levels are assumed to be zero. For the machining operations, the cutting speed and the feed rate will be taken as the decision variables, and the depth of cut is assumed to be given as an input. The CNC machine can work for a limited number of hours. We produce $r$ equal lots of size $Q_{1}$ and one last lot of size $Q_{2}$, such that $D=r Q_{1}+Q_{2}$. Therefore, the average inventory in a given period, AI, after dropping the part indices $p$ for clarity, can be found as follows.

$$
\begin{aligned}
\mathrm{AI}= & {\left[\frac{r \cdot Q_{1}^{2}}{2 D}\left(1-\frac{D \cdot \sum_{i \in I_{p}} \sum_{j \in J} t_{m_{i j p}}}{\mathrm{MH}}\right)\right.} \\
& \left.+\frac{Q_{2}^{2}}{2 D}\left(1-\frac{D \sum_{i \in I_{p}} \sum_{j \in J} t_{m_{i j p}}}{\mathrm{MH}}\right)\right] \\
& /\left(\frac{r \cdot Q_{1}}{D}+\frac{Q_{2}}{D}\right) \\
= & \frac{r \cdot Q_{1}^{2}+Q_{2}^{2}}{2 D}\left(1-\frac{D \cdot \sum_{i \in I_{p}} \sum_{j \in J} t_{m_{i j p}}}{\mathrm{MH}}\right)
\end{aligned}
$$

We can easily verify that $\mathrm{AI}=\frac{Q}{2}\left(1-\frac{D \cdot \Sigma_{i \in I_{p}} \Sigma_{j \in J} t_{m_{i j p}}}{\mathrm{MH}}\right)$ when $Q_{1}=Q_{2}=Q$.

Under these conditions, we are required to solve lot sizing, tool allocation and machining conditions optimization problems simultaneously to determine the following decision variables:

(i) In what quantities each part will be produced, i.e., determination of lot sizes for parts.

(ii) How tools will be allocated to parts in terms of quantities and allocation scheme, and

(iii) What will be the cutting speed and feed rate for each operation of each part.

Advances in cutting tool materials and designs will increase the cutting speeds at which machining is carried out, consequently reduce the machining time, but the initial tooling cost might be higher. Therefore, we consider a set of alternative cutting tool types for each machining operation, since no one cutting tool type is best for all purposes. Moreover, the same tool may be used in several machining operations, each one with different machining conditions. Furthermore, the total production cost should be expressed in terms of the machining, non-machining and tooling costs in addition to the setup and inventory holding costs. Machining time, $t_{m_{i j p}}$, is the time required to complete a turning operation as given in Gorczyca [5]. Tool life, $T_{i j p}$, is generally defined as the machining time in minutes taken to produce a given wear land for a set of 
machining conditions. The relationship between the tool life and the machining conditions can be expressed as a function of the machining conditions by using an extended form of the Taylor's tool life equation. For the turning operation, a new expression is defined for the machining time to tool life ratio, which is called as the usage rate of tool $j$ in operation $i$ of part $p$, and is denoted by $U_{i j p}$. Consequently, $q_{i j p}=\left\lfloor 1 / U_{i j p}\right\rfloor$.

$$
\begin{aligned}
U_{i j p} & =\frac{t_{m_{i j p}}}{T_{i j p}}=\frac{\left(\pi G_{i p} L_{i p}\right) /\left(12 v_{i j p} f_{i j p}\right)}{T C_{j} /\left(v_{i j p}^{\alpha_{j}} f_{i j p}^{\beta_{j}} d_{i p}^{\gamma_{j}}\right)} \\
& =\frac{\pi G_{i p} L_{i p} d_{i p}^{\gamma_{j}}}{12 T C_{j} v_{i j p}^{\left(1-\alpha_{j}\right)} f_{i j p}^{\left(1-\beta_{j}\right)}}
\end{aligned}
$$

For practical purposes, $q_{i j p}$ must be found in order to instruct either the CNC program or the operator to change tools after a predetermined number of pieces have been machined. Furthermore, all time consuming events except the actual cutting operation are called non-machining time components. Although there might be many distinct nonmachining time components such as tool tuning, workpiece loading/unloading, etc., we consider tool replacing times, $t_{r_{j}}$, and loading times, $t_{l_{j}}$, since they are the only ones that can be expressed as a function of both the machining conditions and alternative operation-tool pairs.

A mathematical formulation of the problem can be as follows:

\section{Minimize}

$$
\begin{aligned}
& \sum_{p \in P} S_{p}\left(r_{p}+s_{p}\right)+\sum_{p \in P} \frac{h_{p}}{2 D_{p}}\left(r_{p} Q_{1_{p}}^{2}+Q_{2_{p}}^{2}\right) \\
& \times\left(1-\frac{D_{p} \sum_{i \in I_{p}} \sum_{j \in J} t_{m_{i j p}}}{\mathrm{MH}}\right) \\
& +\sum_{p \in P} D_{p} C_{o} \sum_{i \in I_{p}} \sum_{j \in J} x_{i j p} t_{m_{i j p}} \\
& +\sum_{p \in P}\left(r_{p}+s_{p}\right) C_{o} \\
& \quad \times \sum_{i \in I_{p}} \sum_{j \in J} x_{i j p}\left(\left(n_{i j p}-1\right) t_{r_{j}}+t_{l_{j}}\right) \\
& +\sum_{p \in P} D_{p} \sum_{i \in I_{p}} \sum_{j \in J} x_{i j p} U_{i j p} C_{t_{j}}
\end{aligned}
$$

Subject to:

- Demand Satisfaction Constraints:

$r_{p} Q_{1_{p}}+Q_{2_{p}}=D_{p}$, for every $p \in P$

$Q_{2_{p}} \leq M s_{p}$, for every $p \in P$

- Machine Hour Availability Constraint:

$$
\begin{aligned}
& \sum_{p \in P} D_{p} \sum_{i \in I_{p}} \sum_{j \in J} x_{i j p} t_{m_{i j p}}+\sum_{p \in P}\left(r_{p}+s_{p}\right) \\
& \quad \times \sum_{i \in I_{p}} \sum_{j \in J} x_{i j p}\left(\left(n_{i j p}-1\right) t_{r_{j}}+t_{l_{j}}\right) \\
& \quad+\sum_{p \in P} t s_{p}\left(r_{p}+s_{p}\right) \leq \mathrm{MH}
\end{aligned}
$$

- Tool Assignment Constraints:

$\sum_{j \in J_{i p}} x_{i j p}=1$, for every $i \in I_{p}, p \in P$

$n_{i j p} \leq M x_{i j p}$, for every $i \in I_{p}, j \in J_{i p}, p \in P$

$x_{i j p} \geq U_{i j p}$, for every $i \in I_{p}, j \in J_{i p}, p \in P$

- Tool Availability Constraints:

$\sum_{p \in P} \sum_{i \in I_{p}} D_{p} x_{i j p} U_{i j p} \leq N_{j}$, for every $j \in J$

- Tool Life Constraints:

$x_{i j p} U_{i j p} q_{i j p} \leq 1$, for every $i \in I_{p}, j \in J, p \in P$

- Machine Power Constraints:

$x_{i j p} C_{m} v_{i j p}^{b} f_{i j p}^{c} d_{i p}^{e} \leq \mathrm{HP}$,

for every $i \in I_{p}, j \in J_{i p}, p \in P$

- Surface Roughness Constraints:

$x_{i j p} C_{s} v_{i j p}^{g} f_{i j p}^{h} d_{i p}^{l} \leq \mathrm{SF}_{i p}$,

for every $i \in I_{p}, j \in J_{i p}, p \in P$

- Non-negativity and Integrality Constraints:

$v_{i j p}, f_{i j p}>0, Q_{1_{p}}, Q_{2_{p}} \geq 0, x_{i j p}, s_{p}=\{0,1\}$

and $n_{i j p}, q_{i j p}, r_{p}$ positive integers for every

$p \in P, i \in I_{p}, j \in J$

In this non-linear MIP formulation, the objective function is composed of setup, inventory holding, machining, non-machining and tooling costs, respec- 
tively. The setup and inventory holding cost calculations are based on a common lot sizing assumption that a new lot is produced when the inventory level drops to the reorder point. If a tool is not fully utilized for machining a part then it can be used for machining other parts. Therefore, we introduce the actual tool usage concept, $U_{i j p}$, as discussed earlier. Moreover the production rate for each part is a function of both the available machine hour and total processing time for each part. We satisfy the demand for each part in the first set of constraints. The second constraint ensures that total time required, which is composed of machining, non-machining and set up time components, does not exceed available machine capacity. The third set of constraints represents the operational constraints which guarantee that each operation is assigned to a single tool type of its candidate tools set. The fourth set of constraints ensures that total tool requirement does not exceed the amount of tools on hand. The fifth set of constraints guarantees that machining time for an operation does not exceed available tool life and finally the last two sets of constraints represent usual machining operation constraints. The surface roughness presents the quality requirement on the operation and the machine power constraint ensures that machine tool operates without being subject to any damage.

\section{Algorithm}

The constraints and the decision variables for lot sizing, tool allocation and machining conditions interact with each other. If we increase either $v_{i j p}$ or $f_{i j p}$, or both, then we can reduce the machining time but this will increase the non-machining time and tooling costs. On the other hand, depending on the lot size and machining conditions, the number of tools required to produce a certain operation might be greater than one. Therefore, we propose a new solution procedure by relaxing the machine hour availability constraint, which can be called a coupling constraint among the parts, to solve these interrelated problems simultaneously. For the reduced problem, we then relax the set of tool availability constraints. In this nested minimization procedure, we first find the optimum machining conditions for every possible operation-tool pair and select the tool that gives the minimum cost by using the single machining operation problem (SMOP) as a key, afterwards we impose the relaxed constraints.

In SMOP, the objective function includes the operating cost due to the machining time and tooling cost subject to the machining and tool life constraints. The following geometric programming (GP) formulation can be written for every possible operation-tool pair: Minimize

$$
\begin{aligned}
\mathrm{SMOP}_{i j p} & =C_{o} t_{m_{i j p}}+C_{t_{j}} U_{i j p} \\
& =C_{1} v_{i j p}^{-1} f_{i j p}^{-1}+C_{2} v_{i j p}^{\left(\alpha_{j}-1\right)} f_{i j p}^{\left(\beta_{j}-1\right)}
\end{aligned}
$$

Subject to:

$C_{t}^{\prime} v_{i j p}^{\left(\alpha_{j}-1\right)} f_{i j p}^{\left(\beta_{j}-1\right)} \leq 1$ (Tool Life Constraint)

$C_{m}^{\prime} v_{i j p}^{b} f_{i j p}^{c} \leq 1$ (Machine Power Constraint)

$C_{s}^{\prime} v_{i j p}^{g} f_{i j p}^{h} \leq 1$ (Surface Roughness Constraint)

$v_{i j p}, f_{i j p}>0$

where,

$C_{1}=\frac{\pi G_{i p} L_{i p} C_{o}}{12}, C_{2}=\frac{\pi G_{i p} L_{i p} d_{i p}^{\gamma_{j}} C_{t_{j}}}{12 \mathrm{TC}_{j}}$

$C_{t}^{\prime}=\frac{\pi G_{i p} L_{i p} d_{i p}^{\gamma_{j}} q_{i j p}}{12 \mathrm{TC}_{j}}, C_{m}^{\prime}=\frac{C_{m} d_{i p}^{e}}{\mathrm{HP}}$,

and $C_{s}^{\prime}=\frac{C_{s} \cdot d_{i p}^{l}}{\mathrm{SF}_{i}}$

Therefore, we have a posynomial GP problem since all coefficients $C_{1}, C_{2}, C_{t}^{\prime}, C_{m}^{\prime}$ and $C_{s}^{\prime}$ are strictly positive, and the resulting degree of difficulty is 2. A more detailed discussion on GP can be found in Bazaraa et al. [2]. Each of the constraints of this primal problem can be either loose or tight at optimality, that creates $2^{3}=8$ different cases and only one of them is feasible at the optimal solution. Therefore, the exact solution for SMOP can be found by solving each of these eight cases at the worst case. When we write the GP-dual formulation for the above problem, the objective function for the dual problem is still non-linear, but three constraints of the dual formulation, namely a normalization and two orthogonality constraints, will be linear equations. Dembo [3] presents different algorithms for 
solving the geometric dual of posynomial programming problems. Since we have a relatively small GP problem, for a given problem instance we can define the analytical characterizations of the dual solution for each of the aforementioned eight cases as discussed in Akturk and Avci [1]. If a dual feasible solution is found for a given problem then the corresponding primal solution can be evaluated in terms of its decision variables, and consequently the primal feasibility of the solution can be checked. At optimality, the corresponding solution should be feasible in both the dual and primal problems, and the objective function value for both problems should be the same. This will provide a lower bound for the tool allocation and machining conditions optimization problem. Consequently, the non-linear MIP formulation with several set of constraints given in Section 2 is polynomially transformed to a much simpler integer programming (IP) formulation as outlined below in step 7.

The steps of the proposed algorithm are given below, whereas a step-by-step illustration is given in the next section in a numerical example.

- Step 1: Determination of Possible Lot Sizes. Let $F=\varnothing$ and $r=1$. Do following while $r \leq D_{p}$.

- Step 1.1: $B_{1}=\left\lfloor D_{p} / r\right\rfloor$ and $F=F \cup\left\{B_{1}\right\}$.

- Step 1.2: $r=r+1$.

- Step 2: Determination of Alternative Production Schedules.Let $k=0$ and $K_{p}=\emptyset$. For every $B_{1} \in F$ do the following.

- Step 2.1: $k=k+1$ and $K_{p}=K_{p} \mathrm{U}\{k\}$.

- Step 2.2: $Q_{1_{k p}}=B_{1}$.

If $D_{p} / B_{1}$ is integer then $r_{k p}=D_{p} / B_{1}$ and $Q_{2_{k p}}=$ 0

Else $B_{2}=D_{p}-\left\lfloor D_{p} / B_{1}\right\rfloor B_{1}$

If $B_{2} \leq B_{1} / 2$ then $r_{k p}=\left\lfloor D_{p} / B_{1}\right\rfloor-1$ and $Q_{2_{k p}}=B_{1}$ $+B_{2}$

Else if $B_{2}>B_{1} / 2$ then $r_{k p}=\left\lfloor D_{p} / B_{1}\right\rfloor$ and $Q_{2_{k p}}$ $=B_{2}$

- Step 3: Tool Allocation and Machining Conditions Optimization.

Determine approximate tool allocations $N_{j p}=$ $N_{j} \sum_{i \in I_{p}} y_{i j p} / \sum_{p \in p} \sum_{i \in I_{p}} y_{i j p}$ for every $j \in J$ and $p \in P$, and solve the following steps 3.1 and 3.2 both for $B=Q_{1_{k p}}$ and for $B=Q_{2_{k p}}$ if $Q_{2_{k p}} \neq 0$, respectively for every alternative $k \in K_{p}$. Initially, $q_{i j p}=$ $\left\lceil B / N_{j p}\right\rceil$ to ensure the feasibility in terms of the tool availability constraints.
- Step 3.1: For every possible operation-tool pair $(i, j)$ where $j \in J_{i p}$ solve SMOP to determine $v_{i j p}$, $f_{i j p}, U_{i j p}$ and consequently $q_{i j p}=\left\lfloor 1 / U_{i j p}\right\rfloor$ and $n_{i j p}$ $=\left\lceil B / q_{i j p}\right\rceil$.

- Step 3.2: In SMOP calculations, we minimize the operating cost comprised of the machining and tooling costs, hence the total manufacturing cost can be decreased while increasing the cost of SMOP due to a possible decrease in non-machining costs. Furthermore, there is a tool contention among the operations due to tool availability constraints. Therefore, we resolve SMOP for every $i \in I_{p}, j \in J_{i p}$ and tool requirement level $m \in 1,2, \ldots, n_{i j p}$, denoted as $\mathrm{SMOP}_{i j p}^{m}$ where $q_{i j p}=\lceil B / m\rceil$, to determine the cost $C_{i j p}^{m}=B\left(\mathrm{SMOP}_{i j p}^{m}\right)+C_{o}\left[(m-1) t_{r_{j}}+t_{l_{j}}\right]$. Find the $(j, m)$ pair giving the minimum $C_{i j p}^{m}$ value. If $\sum_{i \in I_{p}} B U_{i j p}^{m} \leq N_{j p}$ for every $j \in J_{i p}$ and $i \in I_{p}$ then we satisfy the tool availability constraint for this part, otherwise solve the following IP formulation to find $v_{i j p}^{*}, f_{i j p}^{*}, U_{i j p}^{*}$ and $n_{i j p}^{*}$ corresponding to the optimum $x_{i j p}^{*}$ such that $C_{k p}^{1}=C_{i j p}^{*}, R_{j k p}^{1}=Q_{1_{k p}} U_{i j p}^{*}$ and $H_{k p}^{1}=Q_{1_{k p}} t_{m_{i j p}}^{*}+\left(n_{i j p}^{*}-1\right) t_{r_{j}}+t_{l_{j}}$ for $Q_{1_{k p}}$. Minimize

$$
\sum_{i \in I_{p}} \sum_{j \in J} \sum_{m=1}^{n_{i j p}} C_{i j p}^{m} x_{i j p}^{m}
$$

Subject to:

$$
\begin{aligned}
& \sum_{j \in J} \sum_{m=1}^{n_{i j p}} x_{i j p}^{m}=1 \forall i \in I_{p} \\
& \sum_{i \in I_{p}} \sum_{m=1}^{n_{i j p}} m x_{i j p}^{m} \leq N_{j p} \quad \forall j \in J
\end{aligned}
$$

The calculations for $C_{k p}^{2}, R_{j k p}^{2}$ and $H_{k p}^{2}$ will be similar if $Q_{2_{k p}} \neq 0$.

- Step 4: Determination of Parameters for Alternative Production Schedules.For every $k \in K_{p}$ and $p \in P$ find $C_{k p}, H_{k p}$ and $R_{j k p}$ for every $j \in J$, where $s_{k p}$ is $0-1$ variable which is equal to 1 if $Q_{2_{p}}>0$ and 0 otherwise, as follows.

$$
\begin{aligned}
C_{k p}= & r_{k p} C_{k p}^{1}+s_{k p} C_{k p}^{2}+\frac{h_{p}}{2 D_{p}}\left(r_{p} Q_{1_{k p}}^{2}+Q_{2_{k p}}^{2}\right) \\
& \times\left(1-\frac{D_{p} \sum_{i \in I_{p}} \sum_{i \in J} t_{m_{i j p}}}{\mathrm{MH}}\right)+S_{p}\left(r_{k p}+s_{k p}\right)
\end{aligned}
$$


$H_{k p}=r_{k p} H_{k p}^{1}+s_{k p} H_{k p}^{2}+t s_{p}\left(r_{k p}+s_{k p}\right)$

$R_{j k p}=r_{k p} R_{j k p}^{1}+s_{k p} R_{j k p}^{2}$ for all $j \in J$

- Step 5: Lower Bound Check.For every part $p \in P$ find alternatives with minimum costs to calculate the lower bound. If these alternatives satisfy the following machine hour and tool availability constraints, such that $\sum_{p \in P} H_{b p} \leq \mathrm{MH}$ and $\sum_{p \in P} R_{j b p}$ $\leq N_{j}$ for every $j \in J$ where $b=\operatorname{argmin}_{k}\left\{C_{k p}\right\}$, then the solution is optimum, STOP.

- Step 6: Preprocessing.

- Step 6.1: Elimination of Dominated Alternatives. Eliminate any dominated alternative $t \in K_{p}$ for which $\exists k \in K_{p}$ such that following conditions are satisfied: $C_{t p} \geq C_{k p}, H_{t p} \geq H_{k p}$, and $R_{j t p} \geq R_{j k p}$ for every $j \in J$.

- Step 6.2: Elimination of Infeasible Alternatives. Compute $R_{\min j p}=\min _{k \in K_{p}}\left\{R_{j k p}\right\}, R_{\min j}=$ $\sum_{p \in P} R_{\min j p}, H_{\min p}=\min _{k \in K}\left\{H_{k p}\right\}$ and $H_{\min }=$ $\sum_{p \in P} H_{\min p}$. If either $R_{\min j}>N_{j}$ or $H_{\min }>\mathrm{MH}$ then the initial part loading solution is not attainable. Otherwise, eliminate any alternative $t \in K_{p}$ and $p \in$ $P$ for which either $\exists j \in J$ such that $R_{j t p}>N_{j}-$ $R_{\min j}+R_{\min j p}$ or $H_{t p}>\mathrm{MH}-H_{\min }+H_{\min p}$.

- Step 7: Solve the following 0-1 IP to find the optimum combination of alternatives.

Minimize

$$
\sum_{p \in P} \sum_{k \in K_{p}} C_{k p} z_{k p}
$$

Subject to:

$\sum_{k \in K_{p}} z_{k p}=1$ for every $p \in P$

$\sum_{p \in P} \sum_{k \in K_{p}} R_{j k p} z_{k p} \leq N_{j}$ for every $j \in J$

$\sum_{p \in P} \sum_{k \in K_{p}} H_{k p} z_{k p} \leq \mathrm{MH}$

$z_{k p} \in\{0,1\}$ for every $p \in P, k \in K_{p}$

In the above formulation the first set of constraints ensures that for each part $p$ exactly one alternative is selected. By the second set of constraints, it is guaranteed that tool availability constraint is not violated for any tool type, and finally the third constraint ensures that the solution does not exceed available machine hour.
The first four steps of the above algorithm is executed for every $p \in P$. In step 1 , we determine the possible lot sizes for possible setups $r \in$ $\left\{1,2,3, \ldots, D_{p}\right\}$ that satisfy the demand satisfaction constraints, and keep these lot sizes in a set $F$. In step 2, we create alternative production schedules for each lot size $B_{1} \in F$. We first check if it exactly divides the demand, since in this case we can satisfy the demand by producing $r=D_{p} / B_{1}$ lots of size $B_{1}$. Otherwise, we produce a last lot of $Q_{2_{k p}}$ if there is a remaining unsatisfied demand $B_{2}$. If the remnant units are greater than one-half of the equal lot size, i.e., $B_{2}>B_{1} / 2$, then we produce them as a separate lot of size $B_{2}$. If they are less than or equal to one-half, i.e., $B_{2} \leq B_{1} / 2$, then we combine them with the last equal lot processed. According to these decisions, we set the size of equal lots to $Q_{1_{k p}}$, the size of last lot to $Q_{2_{k p}}$ and the number of equal lots to $r_{k p}$. In step 3 , the available tools are initially divided among parts in accordance to their requirements of each type, and we determine optimum tool allocation and machining conditions for the lot sizes of $Q_{1_{k p}}$ and $Q_{2_{k p}}$, if $Q_{2_{k p}}>0$, assuming that there are alternative tools for each operation and limited tools available on hand. Initially a lower bound solution is found by relaxing the set of tool availability constraints. If any constraint is violated by the lower bound solution then an IP formulation is solved in step 3.2. In step 4, for every alternative $k$ of part $p$, using the cost, tool and machine hour requirements for $Q_{1_{k p}}$ and $Q_{2_{k p}}$, we determine total cost, total tool and machine hour requirements. At the end of first four steps we generate a set of alternatives for all parts. We find a lower bound solution by selecting the alternative with minimum cost for every part $p \in P$, and check its feasibility in step 5 . If this solution does not violate machine hour and tool availability constraints then the solution is optimum. Otherwise, we preprocess the available alternatives

Table 1

Tooling information

\begin{tabular}{lllll}
\hline Tool no. & $t_{r_{j}}$ & $t_{l_{j}}$ & $N_{j}$ & $C_{t_{j}}$ \\
\hline 1 & 0.91 & 1.06 & 5 & 4.67 \\
2 & 0.91 & 1.18 & 3 & 4.05 \\
3 & 0.82 & 1.34 & 4 & 4.35 \\
4 & 0.96 & 1.30 & 3 & 4.99 \\
\hline
\end{tabular}


Table 2

Possible operation-tool assignments for parts

\begin{tabular}{|c|c|c|c|c|c|c|c|c|c|}
\hline \multirow[t]{2}{*}{ Operation } & \multicolumn{4}{|c|}{ Part 1} & \multicolumn{5}{|c|}{ Part 2} \\
\hline & 1 & 2 & 3 & 4 & 1 & 2 & 3 & 4 & 5 \\
\hline Tool 1 & 0 & 1 & 0 & 1 & 1 & 0 & 1 & 1 & 0 \\
\hline Tool 2 & 1 & 0 & 1 & 0 & 0 & 1 & 0 & 1 & 1 \\
\hline Tool 3 & 0 & 1 & 0 & 0 & 1 & 1 & 1 & 1 & 0 \\
\hline Tool 4 & 0 & 1 & 0 & 0 & 1 & 0 & 1 & 0 & 0 \\
\hline
\end{tabular}

to reduce the search space, and the dominated ones are eliminated in step 6.1. For any part $p$, an alternative $t \in K_{p}$ is dominated, if there exists another alternative $k \in K_{p}$ that is no worse than alternative $t$ in terms of cost, machine hour and tool requirements. In step 6.2, we eliminate the alternatives exceeding either tool or machine hour availability limits. Finally in step 7, over the set of remaining non-dominated alternatives, we construct and solve an IP formulation to find the optimum solution.

\section{A numerical example}

In this example problem, there are two parts that require four cutting tools. The input data related to the tools and parts are given in Tables 1-4.

Data related to all possible alternative production schedules obtained at the end of first four steps are summarized in Table 5. The detailed cost and time components for alternatives of part 2 are also illustrated in Figs. 1 and 2, respectively, as an example. Although the alternative numbers are discrete, we connect these discrete points with straight lines to indicate the expected curve of each cost and time component when we decrease the lot sizes from alternative \#1 to alternative \#13.

We skip step 5 in order to explain the remaining steps. In step 6.1 we eliminate dominated alterna- tives $5,6,7,8,9,10,11,12$ and 13 for part 1 and 4 , $5,6,7,8,9,10,11$ and 12 for part 2. Among remaining alternatives, we eliminate alternatives 14 of part 1 and 13 of part 2 due to tool availability in step 6.2. The alternative 1 of part 1 is not dominated, although it is more expensive and requires more machine hours than the remaining ones, because it needs the minimum number of tools. Finally, in step 7 , we solve the following 0-1 IP formulation to find the optimum combination of alternatives.

$\begin{array}{ll}\text { Min } \quad & 178.1 z_{11}+132.2 z_{21}+133.8 z_{31}+ \\ & 126.2 z_{41}+137.5 z_{12}+124.0 z_{22}+ \\ & 111.2 z_{32} \\ \text { s.t. } \quad & z_{11}+z_{21}+z_{31}+z_{41}=1 \\ & z_{12}+z_{22}+z_{32}=1 \\ & 1.0 z_{11}+2.2 z_{21}+3.3 z_{31}+0.3 z_{41}+ \\ & 1.0 z_{12}+0.1 z_{22}+0.1 z_{32} \leq 5 \\ & 0.1 z_{11}+0.2 z_{21}+0.3 z_{31}+0.4 z_{41}+ \\ & 2.1 z_{12}+2.1 z_{22}+0.2 z_{32} \leq 3 \\ & 2.1 z_{12}+2.1 z_{22}+0.2 z_{32} \leq 4 \\ & 0.1 z_{11}+0.1 z_{21}+0.1 z_{31}+0.1 z_{41}+ \\ & 1.1 z_{12}+0.1 z_{22}+0.2 z_{32} \leq 3 \\ & 273.1 z_{11}+193.3 z_{21}+184.8 z_{31}+ \\ & 193.2 z_{41}+152.2 z_{12}+163.5 z_{22}+ \\ & 176.2 z_{32} \leq 1000\end{array}$

The solution of the above problem is as follows: $z_{41}=z_{32}=1$ giving optimum cost of 237.4. This solution suggests to select alternatives 4 and 3 for parts 1 and 2, respectively. Alternative 4 of part 1 proposes production of 3 lots of size 12 and one lot of size 14, whereas alternative 3 of part 2 corresponds to 3 equal lots of size 15 . The detailed machining parameters and tool allocations of parts 1 and 2 are given in Tables 6-8. On the other hand, if we solve the lot sizing and tool management problems separately using a two-level approach, then alternative 2 will be the best solution for both of the

Table 3

Operation data for parts

\begin{tabular}{|c|c|c|c|c|c|c|c|c|c|}
\hline \multirow[t]{2}{*}{ Operation } & \multicolumn{4}{|l|}{ Part 1} & \multicolumn{5}{|l|}{ Part 2} \\
\hline & 1 & 2 & 3 & 4 & 1 & 2 & 3 & 4 & 5 \\
\hline $\mathrm{SF}_{i p}$ & 336 & 335 & 342 & 167 & 229 & 110 & 308 & 148 & 264 \\
\hline$G_{i p}$ & 1.75 & 1.63 & 2.43 & 2.31 & 1.60 & 1.60 & 1.58 & 2.44 & 1.93 \\
\hline$L_{i p}$ & 6.60 & 5.20 & 6.10 & 5.10 & 6.50 & 6.50 & 5.10 & 6.50 & 5.70 \\
\hline
\end{tabular}


Table 4

Data for numerical example

\begin{tabular}{lllll}
\hline Part & $D_{p}$ & $h_{p}$ & $S_{p}$ & $t s_{p}$ \\
\hline 1 & 50 & 1.4 & 7.0 & 5.5 \\
2 & 45 & 1.5 & 7.5 & 8.5 \\
\hline
\end{tabular}

$\mathrm{MH}=1000 \min , C_{o}=\$ 0.5 / \mathrm{min}$, and $\mathrm{HP}=5 \mathrm{hp}$.

parts giving a total cost of 256.2. Thus, we decrease the total production cost by $7.9 \%$ by reducing the lot sizes.

\section{Computational results}

The algorithm presented in Section 4 were coded in $\mathrm{C}$ language and compiled with Gnu $\mathrm{C}$ compiler. The IP formulations in steps 3.2 and 7 were solved by using callable library routines of CPLEX MIP solver on a Sparcstation 10 under SunOS 5.4. In this section, the efficiency of the proposed algorithm were tested by comparing the total cost found by the algorithm with the costs found by using a traditional two-level approach. In a two-level approach, lot sizing and machining economics decisions are given independently. In the first level lot size is determined by minimizing the sum of setup and inventory holding costs and this lot size is taken as an input by the second level to find the tool management decisions. There are six experimental factors that can affect the efficiency of our algorithm, which are listed in Table 9. Both the number of parts and demand level are most likely to affect the computation times and production costs. The third factor is taken as S/I ratio such that the setup cost for each part is equal to the S/I ratio times the inventory holding cost. The fourth and fifth factors specify the cutting tool cost for each tool type and the tightness of the tool availability constraints, respectively. The number of available tools on hand is taken as $70 \%$ and $90 \%$ of the upper bound on the available tools for each tool type at low and high levels, respectively. The sixth factor determines the assignment matrix, i.e., random or clustered. At the random level, each cutting tool type can be assigned to a candidate tool set of each operation with an equal probability. But in the clustered case the last operation of each part is taken to be finishing operation whereas the remaining operations to be roughing operations. Since there are six factors and two levels, our experiment is $2^{6}$ full-factorial design, corresponding to 64 combinations. The number of replications for each combination is taken as 5, giving 320 different randomly generated runs.

Other variables were treated as fixed parameters and generated as follows:

- System related parameters, $C_{0}=\$ 0.5 / \mathrm{min}, \mathrm{HP}$ $=5$ h.p., and $\mathrm{MH}=60000 \mathrm{~min}$.

- Operation related parameters, $G_{i p}$ and $L_{i p}$ were selected randomly from the interval $\mathrm{UN} \sim[1.5,2.5]$

Table 5

Alternative production schedules

\begin{tabular}{|c|c|c|c|c|c|c|c|c|c|c|}
\hline \multirow[t]{2}{*}{$k$} & \multicolumn{5}{|c|}{ Part 1} & \multicolumn{5}{|c|}{ Part 2} \\
\hline & $Q_{1_{k p}}$ & $Q_{2_{k p}}$ & $r_{k p}$ & $C_{k p}$ & $H_{k p}$ & $Q_{1_{k p}}$ & $Q_{2_{k p}}$ & $r_{k p}$ & $C_{k p}$ & $H_{k p}$ \\
\hline 1 & 50 & 0 & 1 & 178.1 & 273.1 & 45 & 0 & 1 & 137.5 & 152.2 \\
\hline 2 & 25 & 0 & 2 & 132.2 & 193.3 & 22 & 23 & 1 & 124.0 & 163.5 \\
\hline 3 & 16 & 18 & 2 & 133.8 & 184.8 & 15 & 0 & 3 & 111.2 & 176.2 \\
\hline 4 & 12 & 14 & 3 & 126.2 & 193.2 & 11 & 12 & 3 & 120.3 & 189.4 \\
\hline 5 & 10 & 0 & 5 & 132.2 & 199.0 & 9 & 0 & 5 & 129.6 & 204.0 \\
\hline 6 & 9 & 5 & 5 & 142.1 & 209.2 & 8 & 5 & 5 & 140.9 & 218.7 \\
\hline 7 & 8 & 10 & 5 & 142.0 & 209.2 & 7 & 10 & 5 & 140.9 & 218.7 \\
\hline 8 & 7 & 8 & 6 & 151.4 & 219.4 & 6 & 9 & 6 & 151.7 & 233.4 \\
\hline 9 & 6 & 8 & 7 & 161.2 & 229.7 & 5 & 0 & 9 & 173.4 & 262.8 \\
\hline 10 & 5 & 0 & 10 & 180.7 & 250.1 & 4 & 5 & 10 & 196.4 & 292.1 \\
\hline 11 & 4 & 6 & 11 & 201.2 & 270.5 & 3 & 0 & 15 & 241.9 & 350.8 \\
\hline 12 & 3 & 2 & 16 & 252.1 & 321.6 & 2 & 3 & 21 & 322.4 & 452.1 \\
\hline 13 & 2 & 0 & 25 & 334.2 & 403.4 & 1 & 0 & 45 & 584.1 & 778.9 \\
\hline 14 & 1 & 0 & 50 & 590.9 & 653.6 & & & & & \\
\hline
\end{tabular}




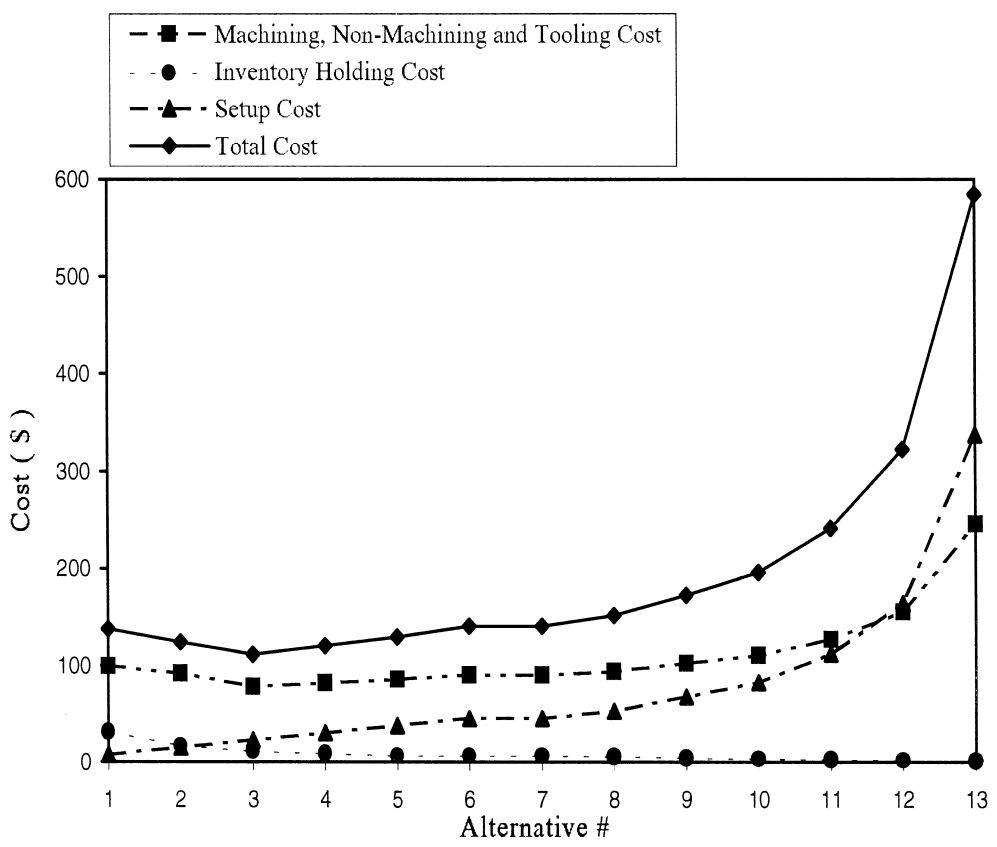

Fig. 1. The detailed analysis of cost components for Part 2.

and $\mathrm{UN} \sim[5,7]$ respectively, where $\mathrm{UN}$ stands for the uniform distribution.

- Number of operations per part UN [3, 5].
- An upper bound on the available number of tools for each tool type were taken as a function of the factors $\mathrm{A}$ and $\mathrm{B}$, namely part number and de-

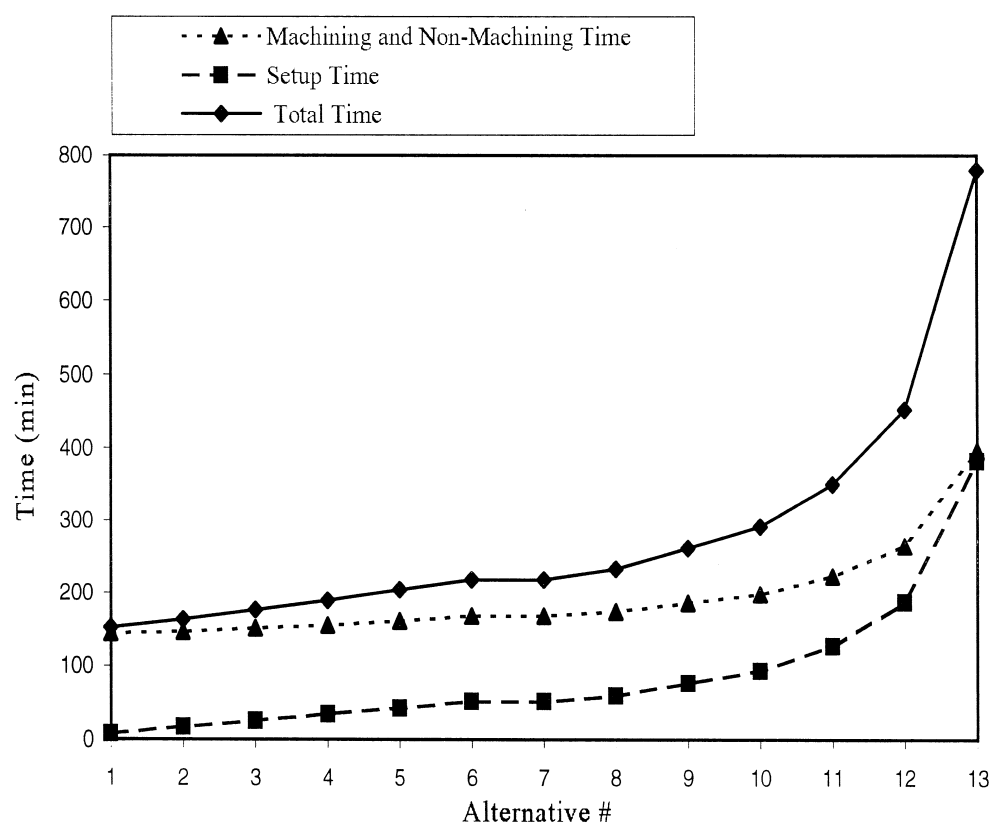

Fig. 2. The detailed analysis of time components for Part 2. 
Table 6

Optimum tool allocation for the equal lots of Part 1

\begin{tabular}{lllllll}
\hline Operation no. & Tool no. & $n_{i j p}$ & $v_{i j p}$ & $f_{i j p}$ & $t_{m_{i j p}}$ & $U_{i j p}$ \\
\hline 1 & 2 & 1 & 273.4 & 0.023 & 0.44 & 0.033 \\
2 & 4 & 1 & 229.3 & 0.021 & 0.87 & 0.035 \\
3 & 2 & 1 & 249.5 & 0.016 & 1.77 & 0.068 \\
4 & 1 & 1 & 323.6 & 0.007 & 3.00 & 0.083 \\
\hline
\end{tabular}

mand level. In the low part number case, tool availability was 50 and 200 for low and high demand levels, respectively, and similarly in high part number case, it was 150 and 600 for low and high demand levels, respectively.

- The values of $\mathrm{SF}_{i p}$ and $d_{i p}$ were related with the assignment matrix. For random assignment matrix, $\mathrm{SF}_{i p}=\mathrm{UN} \sim[30,500]$ and $d_{i p}=\mathrm{UN} \sim[0.025$, $0.3]$. In the clustered case, there were two types of operations, namely roughing and finishing. For roughing operations, $\mathrm{SF}_{i p}=\mathrm{UN} \sim[300,500]$ and $d_{i p}=\mathrm{UN} \sim[0.2,0.3]$, and for the finishing operation, $\mathrm{SF}_{i p}=\mathrm{UN} \sim[30,70]$ and $d_{i p}=\mathrm{UN} \sim[0.025$, 0.075].

- There are 10 different cutting tool types for which $t_{r_{j}}=U N \sim[0.75,1]$ and $t_{l_{j}}=U N \sim[1,1.5]$, and their technological exponents were given in Table 10 .

- Inventory holding cost for each part, $h_{p}$, was selected randomly from the interval $\mathrm{UN} \sim[1,2]$. Furthermore, the setup time, $t s_{p}=(\mathrm{S} / \mathrm{I}$ ratio $) . \mathrm{UN} \sim$ $[1,2]$ and setup cost, $S_{p}=(\mathrm{S} / \mathrm{I}$ ratio $) \cdot h_{p}$.

In a two-level approach, a decision made at the lot sizing level without considering its impact on the tool management problem can lead to infeasible or inferior results when we consider both the constraints and parameters of the tool management problem. In fact, in our experimental design 72 infeasible cases were observed among 320 randomly generated problems, that is approximately $22.5 \%$ of all prob-

Table 7

Optimum tool allocation for the last lot of Part 1

\begin{tabular}{lllllll}
\hline Operation no. & Tool no. & $n_{i j p}$ & $v_{i j p}$ & $f_{i j p}$ & $t_{m_{i j p}}$ & $U_{i j p}$ \\
\hline 1 & 2 & 1 & 273.4 & 0.023 & 0.44 & 0.033 \\
2 & 4 & 1 & 229.3 & 0.021 & 0.87 & 0.035 \\
3 & 2 & 1 & 249.5 & 0.016 & 1.77 & 0.068 \\
4 & 1 & 1 & 310.1 & 0.006 & 3.14 & 0.071 \\
\hline
\end{tabular}

Table 8

Optimum tool allocation for Part 2

\begin{tabular}{lllllll}
\hline Operation no. & Tool no. & $n_{i j p}$ & $v_{i j p}$ & $f_{i j p}$ & $t_{m_{i j p}}$ & $U_{i j p}$ \\
\hline 1 & 4 & 1 & 300.4 & 0.035 & 0.24 & 0.020 \\
2 & 3 & 1 & 470.8 & 0.007 & 1.06 & 0.067 \\
3 & 4 & 1 & 230.5 & 0.019 & 1.52 & 0.037 \\
4 & 1 & 1 & 435.4 & 0.016 & 2.07 & 0.041 \\
5 & 2 & 1 & 257.5 & 0.012 & 2.93 & 0.065 \\
\hline
\end{tabular}

lems. Among these 72 cases, two cases were due to the machine hour violation while remaining 70 cases were due to the tool availability restriction. We summarize overall results of the proposed joint approach along with the minimum, average and maximum values for total production costs and computation times in Table 11. It should be noted that these cost values include all of the production related costs, namely machining, non-machining, tooling, setup and inventory holding costs. In the same table we also presented percent improvements in cost terms obtained over 248 comparable cases, where the twolevel approach found a feasible solution. Among these 248 cases, the maximum improvement of $19.11 \%$ occurred for the case $\left(\begin{array}{llllll}0 & 1 & 1 & 1 & 1 & 1\end{array}\right)$, where zero and one correspond to the low and high levels of each factor, respectively. Furthermore, we improve the total cost by an average of $6.79 \%$ over the two-level approach. A paired- $t$ test was applied to the total cost terms found by the two methods to test the statistical significance of their difference. We found that $t$-value was 11.65 and the cost values were different with $p \leq 0.000$ significance. As we pointed out before, the two-level approach resulted in 72 infeasible solutions among 320 problems, however these infeasible cases were the ones that would increase the average improvement beyond $6.79 \%$ if

Table 9

Experimental factors

\begin{tabular}{llll}
\hline Factors & Definition & Low & High \\
\hline A & Number of parts & 25 & 100 \\
B & Demand & UN $\sim[30,50]$ & UN $\sim[100,200]$ \\
C & S /I ratio & 3 & 10 \\
D & Tooling cost & UN $\sim[3,4]$ & UN $\sim[9,10]$ \\
E & Tool availability & $70 \%$ & $90 \%$ \\
F & Assignment matrix & Random & Clustered \\
\hline
\end{tabular}


Table 10

Technological exponents and coefficients of the available tools

\begin{tabular}{lllllllllllll}
\hline T\# & $\alpha$ & $\beta$ & $\gamma$ & $C_{j}$ & $b$ & $c$ & $e$ & $C_{m}$ & $g$ & $h$ & $l$ & $C_{s}$ \\
\hline$T_{1}$ & 4.0 & 1.40 & 1.16 & 40960000 & 0.91 & 0.78 & 0.75 & 2.394 & -1.52 & 1.004 & 0.25 & 204620000 \\
$T_{2}$ & 4.3 & 1.60 & 1.20 & 37015056 & 0.96 & 0.70 & 0.71 & 1.637 & -1.60 & 1.005 & 0.30 & 259500000 \\
$T_{3}$ & 3.7 & 1.30 & 1.10 & 13767340 & 0.90 & 0.75 & 0.72 & 2.315 & -1.45 & 1.015 & 0.25 & 202010000 \\
$T_{4}$ & 3.7 & 1.28 & 1.05 & 11001020 & 0.80 & 0.75 & 0.70 & 2.415 & -1.63 & 1.052 & 0.30 & 205740000 \\
$T_{5}$ & 4.1 & 1.26 & 1.05 & 48724925 & 0.80 & 0.77 & 0.69 & 2.545 & -1.69 & 1.005 & 0.40 & 204500000 \\
$T_{6}$ & 4.1 & 1.30 & 1.10 & 57225273 & 0.87 & 0.77 & 0.69 & 2.213 & -1.55 & 1.005 & 0.25 & 202220000 \\
$T_{7}$ & 3.7 & 1.30 & 1.05 & 13767340 & 0.83 & 0.75 & 0.73 & 2.321 & -1.63 & 1.015 & 0.30 & 203500000 \\
$T_{8}$ & 3.8 & 1.20 & 1.05 & 23451637 & 0.88 & 0.83 & 0.72 & 2.321 & -1.55 & 1.016 & 0.18 & 213570000 \\
$T_{9}$ & 4.2 & 1.65 & 1.20 & 56158018 & 0.90 & 0.78 & 0.65 & 1.706 & -1.54 & 1.104 & 0.32 & 211825000 \\
$T_{10}$ & 3.8 & 1.20 & 1.05 & 23451637 & 0.81 & 0.75 & 0.72 & 2.298 & -1.55 & 1.016 & 0.18 & 203500000 \\
\hline
\end{tabular}

the two-level approach had found comparable feasible results. This fact can be easily observed in Table 12, where we presented the number of infeasible cases and minimum, average and maximum improvement percentages for the most significant two factors on improvements. Our computational experiments on a set of randomly generated problems indicate that the use of proposed integrated approach offers substantial cost savings over the traditional approach of solving these problems separately. The magnitude of savings is dependent on the system parameters.

We have solved a reasonable size of problems with the maximum of 100 parts, each requiring 4 different operations, and 5 alternative cutting tool types for each operation on the average. The maximum computation time to find an optimum solution was $226.9 \mathrm{~s}$, whereas the average computation time was approximately one minute for the joint approach. Since the computation time depends on the code of the program, the specifications and the configuration of the computer system, we can also indi-

Table 11

Overall results of the experimental design

\begin{tabular}{lcrll}
\hline & \multicolumn{1}{l}{ Min. } & \multicolumn{1}{l}{ Avg. } & \multicolumn{1}{l}{ Max. } & Out of \\
\hline Joint total cost (\$) & 1798.0 & 13310.62 & 49527.9 & 320 \\
$\begin{array}{l}\text { Joint } \\
\text { computation }\end{array}$ & 5.66 & 63.40 & 226.90 & 320 \\
$\begin{array}{l}\text { time (s) } \\
\begin{array}{l}\text { Two-level } \\
\text { computation }\end{array}\end{array}$ & 0.01 & 0.87 & 10.36 & 320 \\
$\begin{array}{l}\text { time (s) } \\
\text { Improvement (\%) }\end{array}$ & 0.74 & 6.79 & 19.11 & 248 \\
\hline
\end{tabular}

cate the computational requirements of the proposed algorithm in terms of the number of operations required to find an optimum solution. Let's look at the following example with 10 parts, each requiring 5 different operations, 2 alternative cutting tool types for each operation out of 5 cutting tool types, and the demand for each part is 500 for a given period. Consequently, there are 43 alternative production schedules for each part, and the number of equal lot size alternatives is equal to 12 out of 43 schedules. Therefore, the SMOP formulation will be solved $(43+31) \cdot(10 \cdot 5 \cdot 2)=7400$ times to determine the optimum machining conditions. If all of these production schedules are both feasible and nondominated after the lower bound check and preprocessing then we have to solve an IP formulation with 430 binary variables and 16 constraints to find the optimum combination of alternatives.

We also applied a two-way analysis of variance (ANOVA) test on the performance measures of total cost, computation time and percent improvements. The $F$ values and significance levels $(p)$ for these performance measures against six factors were given in Table 13. As it was expected, all of the factors

Table 12

Percent improvements and the number of infeasible cases

\begin{tabular}{lll}
\hline $\begin{array}{l}\text { Demand } \\
\text { level }\end{array}$ & S/I ratio & \\
\cline { 2 - 3 } & Low (min., avg., max.) & High (min., avg., max.) \\
\hline Low & $(0.74,1.63,3.27)$ & $(2.78,6.68,12.23)$ \\
& No infeasible cases & No infeasible cases \\
High & $(7.32,10.55,15.57)$ & $(5.15,13.08,19.11)$ \\
& 28 Infeasible cases & 44 Infeasible cases \\
\hline
\end{tabular}


Table 13

$F$ values and significance levels $(p)$ for ANOVA results

\begin{tabular}{|c|c|c|c|c|c|c|}
\hline \multirow[t]{2}{*}{ Factors } & \multicolumn{2}{|c|}{ Total cost } & \multicolumn{2}{|c|}{ Comp. time } & \multicolumn{2}{|c|}{ Improvement } \\
\hline & $F$ & $p$ & $F$ & $p$ & $F$ & $p$ \\
\hline A & 19013.5 & 0.000 & 1580.1 & 0.000 & 4.0 & 0.046 \\
\hline B & 15317.6 & 0.000 & 3.5 & 0.059 & 1048.5 & 0.000 \\
\hline $\mathrm{C}$ & 689.2 & 0.000 & 0.1 & 0.755 & 598.4 & 0.000 \\
\hline $\mathrm{D}$ & 439.2 & 0.000 & 6.7 & 0.010 & 61.8 & 0.000 \\
\hline $\mathrm{E}$ & 0.1 & 0.871 & 38.3 & 0.000 & 8.1 & 0.005 \\
\hline $\mathrm{F}$ & 55.7 & 0.000 & 166.8 & 0.000 & 141.0 & 0.000 \\
\hline
\end{tabular}

except the fifth one, tool availability, were significant for the total production cost with $p \leq 0.000$. Among these factors A and B directly affect the amount to be produced, hence total cost of production whereas the third and fourth factors affect the setup and tooling cost components of the total production cost, respectively. Finally, the sixth factor affects the total cost of production due to the tool allocation and consequently machining conditions decisions. The ANOVA results for the computation time of our algorithm has shown that the most important factors on computation times were the factors $\mathrm{A}, \mathrm{E}$ and $\mathrm{F}$ with $p \leq 0.000$ significance. The factor A directly affects the size of the problem, whereas the factor E constrains the number of tools on hand. The significance of factor $\mathrm{F}$, assignment matrix, depends on the fact that in the clustered case the machining conditions and tool allocation optimization problem is decomposed into two separate problems for roughing and finishing operations, which reduces the number of possibilities. All of the factors were significant on the percent improvements, which also indicated the advantage of the proposed joint approach over a two-level approach.

\section{Conclusions}

We propose a new solution procedure for solving the lot sizing and tool management problems simultaneously to minimize the total production cost. For this purpose, the lot sizing problem is integrated to the machining conditions selection and tool allocation problem to prevent any infeasibility that may occur due to tool and machine hour availability limitations. Most of the lot sizing and tool manage- ment approaches solve these two problems independently using a two-level approach. The following justification seems to be prevalent for not evaluating the lot sizing and tool management problems, jointly. The lot sizing problem is considered a planning decision and is assumed to be solved at a higher level in an organization than is the tool management problem. The tool management problem is considered a low-level, detailed decision problem that should be solved after the lot sizing problem. Unfortunately, the interface between these two problems is critical as discussed in Section 5 and these two problems cannot be viewed in isolation. In the twolevel approach, lot sizes are predetermined prior to the tool management. This might create empty feasible solution spaces and otherwise unnecessarily limit the number of alternatives possible for the tool management problem. Although the computational price of the two-level approach is less than the proposed joint approach, the joint approach dominates and gives much better results than any fixed lot size approach due to the increased solution flexibility. As a final point, an effective tool management is a major requirement for the implementation of an FMS, hence the CNC machine tools as stated by several authors. In the automated environments, sophisticated computerized decision making tools are needed for effective operation and control of the system. In this respect, this study can be considered as a part of the fully automated process planning system.

\section{Acknowledgements}

The authors would like to thank the two anonymous referees for their helpful comments and suggestions on improving the format and contents of the paper. This work was supported in part by the Scientific and Technical Research Council of Turkey under grant MISAG-111.

\section{References}

[1] M.S. Akturk, S. Avci, Tool allocation and machining conditions optimization for CNC machines, European Journal of Operational Research 94 (1996) 335-348.

[2] M.S. Bazaraa, H.D. Sherali, C.M. Shetty, Nonlinear Pro- 
gramming Theory and Algorithms, 2nd edn., Wiley, New York, 1993.

[3] R.S. Dembo, Second order algorithms for the posynomial geometric programming dual: Part I. Analysis, Mathematical Programming 17 (1979) 156-175.

[4] B. Gopalakrishnan, F. Al-Khayyal, Machine parameter selection for turning with constraints: an analytical approach based on geometric programming, International Journal of Production Research 29 (1991) 1897-1908.

[5] F.E. Gorczyca, Application of Metal Cutting Theory, Industrial Press, 1987.

[6] A.E. Gray, A.S. Seidmann, K.E. Stecke, A synthesis of decision models for tool management in automated manufacturing, Management Science 39 (1993) 549-567.

[7] M. Kalta, B.J. Davies, Product representation for an expert process planning system for rotational components, International Journal of Advanced Manufacturing Technology 9 (1994) 180-187.

[8] R.B. Karadkar, S.S. Pande, Feature based automatic CNC code generation for prismatic parts, Computers in Industry 28 (1996) 137-150.

[9] C.P. Koulamas, Optimal lot sizing and machining economics, Journal of Operational Research Society 41 (1990) 943-952.

[10] C.P. Koulamas, Simultaneous determination of the cutting speed and lot size values in machining systems, European Journal of Operational Research 84 (1995) 356-370.

[11] P. Kouvelis, An optimal tool selection procedure for the initial design phase of a flexible manufacturing system, European Journal of Operational Research 55 (1991) 201210.

[12] S.C. Sarin, C.S. Chen, The machine loading and tool allocation problem in a flexible manufacturing system, International Journal of Production Research 25 (1987) 1081-1094.

[13] M.S. Sodhi, R.G. Askin, S. Sen, A hierarchical model for control of flexible manufacturing systems, Journal of Operational Research Society 45 (1994) 1185-1196.

[14] K.E. Stecke, Formulation and solution of nonlinear integer production planning problems for flexible manufacturing systems, Management Science 29 (1983) 273-288.

[15] F.P. Tan, R.C. Creese, A generalized multi-pass machining model for machining parameter selection in turning, International Journal of Production Research 33 (1995) 1467-1487.

[16] C.S. Tang, E.V. Denardo, Models arising from a flexible manufacturing machine: Part I. Minimization of the number of tool switches, Operations Research 36 (1988) 767-777.

[17] U.A.W. Tetzlaff, A queueing network model for flexible manufacturing systems with tool management, IIE Transactions 28 (1996) 309-317.

[18] D. Veeramani, D.M. Upton, M.M. Barash, Cutting tool management in computer integrated manufacturing, International Journal of Flexible Manufacturing Systems 3 (1992) 237-265.

[19] R.A. Wysk, R.P. Davis, R.M.A. Tanchoco, Machining parameter optimization with lot size considerations, AIIE Transactions 12 (1980) 59-63.

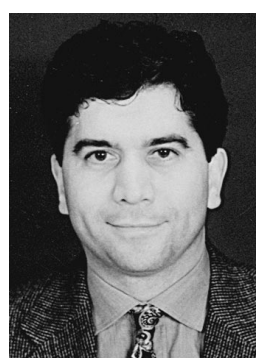

M. Selim Akturk is an Assistant Professor of Industrial Engineering at Bilkent University, Turkey. He holds a Ph.D. in Industrial Engineering from Lehigh University, U.S.A., and B.S.I.E. and M.S.I.E. from Middle East Technical University, Turkey. His current research interests include hierarchical planning of large scale systems, cellular manufacturing systems, production scheduling and advanced manufacturing technologies. Dr. Akturk is a senior member of IIE and member of INFORMS.

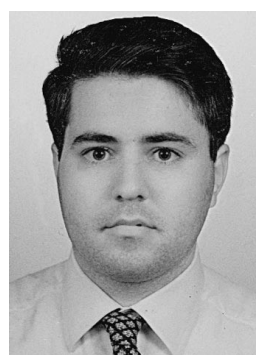

Siraceddin Onen is a senior consultant at International Integration, Cambridge, MA. He received B.S.C.S. and B.S.I.E degrees from Bosphorus University, Turkey and an M.S.I.E. from Bilkent University. His research interests include optimization theory, object oriented design and analysis, object oriented programming languages and management information systems. 